\title{
Growth of Laser Damage in SiO2 under Multiple Wavelength Irradiation
}

M. A. Norton, E. E. Donohue, M. D. Feit, R. P. Hackel, W. G. Hollingsworth, A, M. Rubenchik, M. L. Spaeth

November 1, 2005

Boulder Damage Symposium XXXVII Boulder, CO, United States September 19, 2005 through September 21, 2005 
This document was prepared as an account of work sponsored by an agency of the United States Government. Neither the United States Government nor the University of California nor any of their employees, makes any warranty, express or implied, or assumes any legal liability or responsibility for the accuracy, completeness, or usefulness of any information, apparatus, product, or process disclosed, or represents that its use would not infringe privately owned rights. Reference herein to any specific commercial product, process, or service by trade name, trademark, manufacturer, or otherwise, does not necessarily constitute or imply its endorsement, recommendation, or favoring by the United States Government or the University of California. The views and opinions of authors expressed herein do not necessarily state or reflect those of the United States Government or the University of California, and shall not be used for advertising or product endorsement purposes. 


\title{
Growth of Laser Damage in $\mathrm{SiO}_{2}$ under Multiple Wavelength Irradiation
}

\author{
Mary A. Norton, Eugene E. Donohue, Michael D. Feit, Richard P. Hackel, William G. Hollingsworth, \\ Alexander M. Rubenchik and Mary L. Spaeth \\ Lawrence Livermore National Laboratory, M/S L4592, PO Box 5508, Livermore, CA 94551
}

\begin{abstract}
In laser systems using frequency conversion, multiple wavelengths will be present on optical components. We have investigated the growth of laser initiated damage in fused silica in the presence of multiple wavelengths. In particular, we measured growth at $351 \mathrm{~nm}$ in the presence of $1053 \mathrm{~nm}$ near the threshold of growth for $351 \mathrm{~nm}$ alone. The data shows that the sum fluence determines the onset of growth as well as the growth rate. The measured growth coefficient is consistent with all the energy being delivered at $351 \mathrm{~nm}$. Additionally, we measured growth at $527 \mathrm{~nm}$ in the presence of $1053 \mathrm{~nm}$ near the threshold of growth at $527 \mathrm{~nm}$ alone. In this case, the sum fluence also determines the growth coefficient but the rate is consistent with all the energy being delivered at $1053 \mathrm{~nm}$. We present the measurements and discuss possible reasons for the behavior.
\end{abstract}

Keywords: Laser damage, laser damage growth, laser damage growth threshold, UV fused silica.

\subsection{General}

\section{INTRODUCTION}

Laser systems that use frequency conversion to produce new wavelengths will have optical components with multiple wavelengths present. The lifetime of optics used in laser applications is limited both by laser-initiated damage and by the subsequent growth of the laser-initiated damage. Frequently, it is the growth of laser damage that determines the true operating costs of laser systems, since a damage site that does not grow or grows very slowly will not impede laser operation. Moreover, both laser damage initiation and laser damage growth on the optical exit surfaces dominate the damage events. Previous measurements of laser growth of laser damage sites on the exit surface of fused silica have been obtained at a single wavelength. ${ }^{1,2,3}$

This work focuses on the growth of damage after laser initiation under the simultaneous presence of two wavelengths. In particular, two conditions have been considered. The first condition is growth in the presence of the fundamental wavelength $(1 \omega)$ and the frequency tripled wavelength $(3 \omega)$. These are the two wavelengths that predominate in well designed frequency tripled systems. The second condition is growth in the presence of $1 \omega$ and the frequency doubled wavelength $(2 \omega)$. These are the only two wavelengths in frequency doubled systems. In both sets of experiments the effect of the unconverted wavelength on both growth rate and threshold for growth will be explored.

\subsection{Background}

Our previous measurements of growth of laser damage at the second and third harmonics of a $1 \mu \mathrm{m}$ laser system found a growth threshold of $\sim 5 \mathrm{~J} / \mathrm{cm}^{2}$ for the third harmonic and a growth threshold of $\sim 12 \mathrm{~J} / \mathrm{cm}^{2}$ for the second harmonic. We have focused our attention on determining if the presence of the unconverted fundamental will alter these single wavelength thresholds and in addition whether the growth rates will be affected. In order to define the parameter range of interest we have looked at two specific efficient frequency converters operating in the fluence range of growth of the harmonic wavelengths. The calculations are for a KDP type I, $13 \mathrm{~mm}$ thick doubler and a KDP type I, $11 \mathrm{~mm}$ thick doubler with a $\mathrm{KD}$ *P type II, $9.2 \mathrm{~mm}$ thick tripler, and an input spatially flat top fundamental beam. Temporal wave shape is Gaussian and we have considered three pulse widths: $3.5 \mathrm{~ns}, 10 \mathrm{~ns}$ and $20 \mathrm{~ns}$. The calculated unconverted fundamental fluence is plotted versus the output converted fluence for both cases and the results are shown in figure 1. In both cases, the residual red can span the fluence range of 2 to $12 \mathrm{~J} / \mathrm{cm}^{2}$. Since previous measurements ${ }^{1}$ at $3 \omega$ over a pulse range of $800 \mathrm{ps}$ to $10 \mathrm{~ns}$ found weak dependence of the growth on pulse width, the present measurements will cover a residual $1 \omega$ range of $\sim 2$ to $12 \mathrm{~J} / \mathrm{cm}^{2}$. 


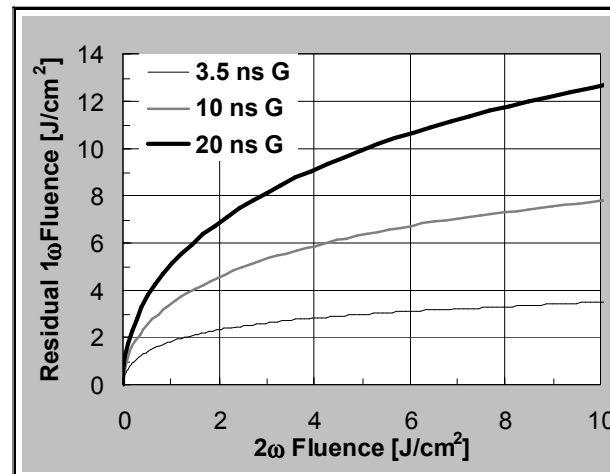

(a)

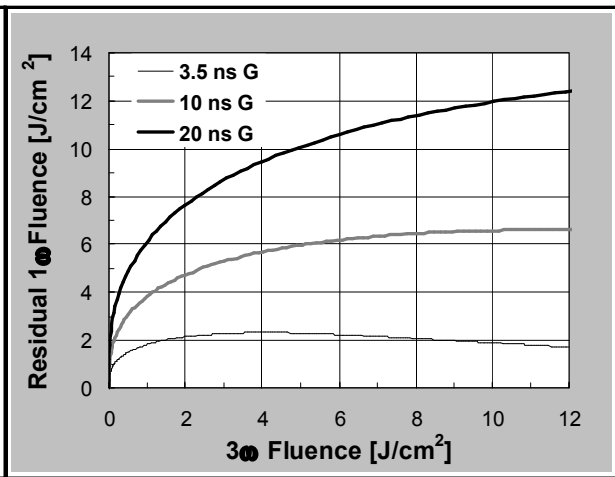

(b)

Figure 1. Calculated residual fundamental versus the harmonic wavelength for two convertors

\subsection{Laser system and testing parameters}

\section{EXPERIMENT}

Laser damage initiation thresholds are typically measured with small beam laser systems where the beam profile is Gaussian and of diameter on the order of 1-mm. To make measurements of laser damage growth that are relevant to large beam areas such as are found on high energy laser systems, it is necessary to use a beam with an area large relative to the initial damage size. At LLNL, a unique laser facility can provide a large area, $1053 \mathrm{~nm}$ beam along with a high rep rate. The laser is the SLAB laser system ${ }^{4}$, a Nd: glass zig-zag slab amplifier, with SBS phase conjugation producing a near diffraction limited $1.053 \mu \mathrm{m}$ output. This is the $1 \omega$ wavelength for these experiments, with $527 \mathrm{~nm}$ and $351 \mathrm{~nm}$ the $2 \omega$ and $3 \omega$ wavelengths respectively. As used for these experiments SLAB provides a $20 \mathrm{~J}, 1053 \mathrm{~nm}$ output pulse, with a $12 \mathrm{nsec}$ FWHM near Gaussian shaped temporal waveform, at a rep rate of $0.5-\mathrm{Hz}$. The rep rate is limited by the data collection rate, as the laser system can be operated at $5 \mathrm{~Hz}$. This is the beam that is formatted and converted into the appropriate wavelengths used in these experiments.

The SLAB 1-micron beam is image relayed onto the experiment table where it is formatted to $1.7 \mathrm{~cm}$ by $1.7 \mathrm{~cm}$ and enters a frequency converter system. The frequency converter is a type I KD*P doubler followed by a type II KD*P tripler. It is the converted and unconverted fundamental wavelength that is then varied in the growth measurements. Though each wavelength follows a different path to the beam combiners before the test chamber the individual path lengths are kept equal to each other within less than a nanosecond. Three important features of the test beams are produced by using this scheme. The depleted fundamental spatial profile tends to have better uniformity than the unconverted beam, the temporal pulse width is longer than the unconverted beam and the relative polarizations are as they would be after exiting the frequency converter. The appropriate pair of wavelengths is spatially filtered before being transported to the sample chamber. The key components of the layout for these experiments with the SLAB laser are shown in figure 2. The sample is located in an image relay plane of the laser and the beam size for all wavelengths on the part is nominally $5 \mathrm{~mm} \times 5 \mathrm{~mm}$. The beams are spatially and temporally overlapped at the sample. The laser is incident at $15^{\circ}$. The fluence of each wavelength is independently controlled with a polarizer/waveplate combination. 


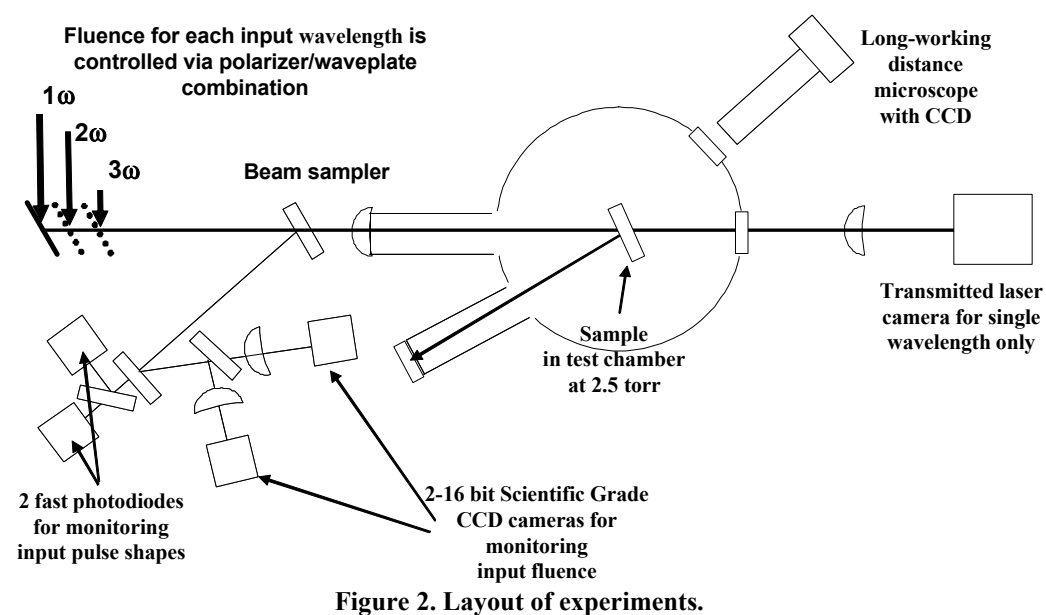

The sample is housed in a stainless steel vacuum chamber, which is located in a class 100 area where samples up to $150 \mathrm{~mm}$ $\mathrm{x} 150 \mathrm{~mm}$ in size are handled during loading. For these tests, conducted in air at 2-10 torr, dry filtered high purity air is used to fill the chamber after it has first been pumped out to vacuum.

Laser beam measurements for both test beams on the part include measurement of the temporal pulse shapes, energy and input \& output beam spatial intensity distributions for each wavelength. Diagnostics to measure the growth include a white light illuminated, long working distance microscope and CCD camera and a scientific grade CCD camera viewing the transmitted light through the site. The workhorses for the growth measurements are two 16-bit scientific-grade CCD cameras that sample the input beams incident on the part. They are calibrated both for energy and for magnification and are used to set and record the fluence on the sample for each shot. In practice, the camera viewing the beam transmitted through the sample is used to locate the starting damage and the input camera is used to set the local fluence in a 1-mm patch surrounding the site. The lateral growth of the damage site can be measured either from the transmitted camera or from the microscope. Typical images of the converted and residual beams and temporal waveforms on the sample for the combined $3 \omega$ and $1 \omega$ tests are shown in figure $3 \mathrm{a}, 3 \mathrm{~b}$ and $3 \mathrm{c}$ respectively. The calculated statistics for the $3 \omega$ beam shown in figure $3 \mathrm{a}$ is a contrast ratio (rms/average) of $18 \%$ over the central $60 \%$ of the beam area. The calculated statistics for the $1 \omega$ beam shown in figure $3 \mathrm{~b}$ is a contrast ratio of $21 \%$ over the central $70 \%$ of the beam area. An overlay of 4 consecutive temporal waveforms is shown in figure $3 \mathrm{c}$, where the average FWHM=11 ns for the $3 \omega$ beam and $18 \mathrm{~ns}$ for the $1 \omega$ beam; also seen in the plot is the relative time of arrival of the two beams with the $1 \omega$ beam arriving approximately $2 \mathrm{~ns}$ before the $3 \omega$ beam. Typical images of the converted and residual beams and temporal waveforms on the sample for the combined $2 \omega$ and $1 \omega$ tests are shown in figure $4 \mathrm{a}, 4 \mathrm{~b}$ and $4 \mathrm{c}$ respectively. The calculated statistics for the $2 \omega$ beam shown in figure $4 \mathrm{a}$ is a contrast ratio of $23 \%$ over the central $65 \%$ of the beam area. The calculated statistics for the $1 \omega$ beam shown in figure $4 \mathrm{~b}$ is a contrast ratio of $10 \%$ over the central $75 \%$ of the beam area. An overlay of 4 consecutive temporal waveforms is shown in figure $4 \mathrm{c}$, where the average $\mathrm{FWHM}=8 \mathrm{~ns}$ for the $2 \omega$ beam and $17 \mathrm{~ns}$ for the $1 \omega$ beam with the $1 \omega$ beam arriving approximately $3.5 \mathrm{~ns}$ before the $2 \omega$ beam. 

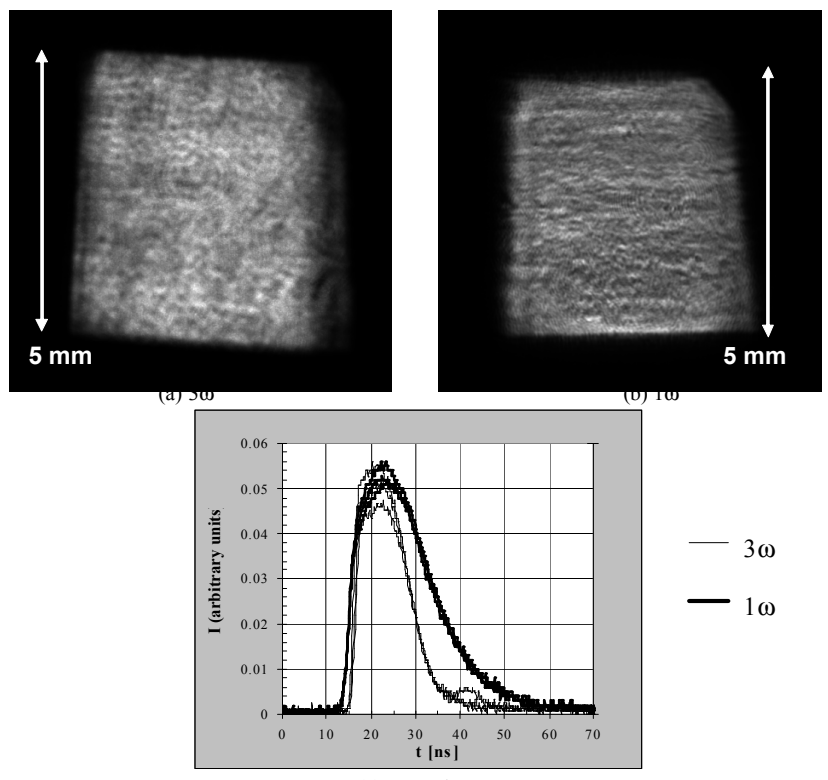

(c) $3 \omega$ and $1 \omega$

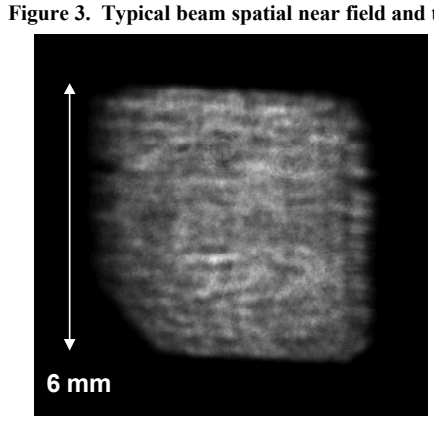

(a) $2 \omega$

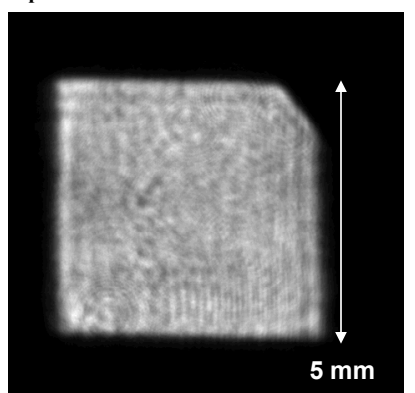

(b) $1 \omega$

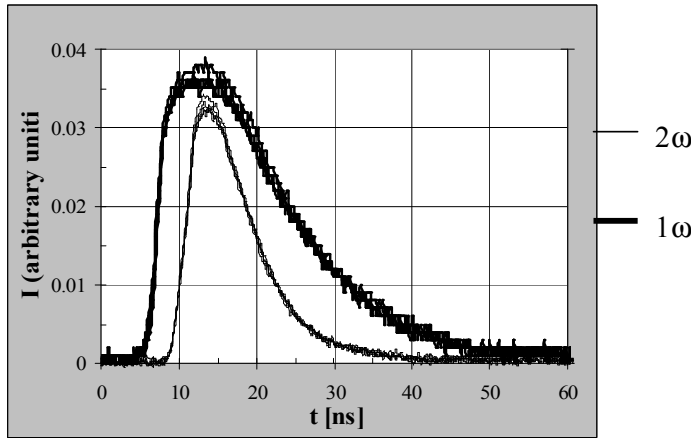

(c) $2 \omega$ and $1 \omega$

Figure 4. Typical beam spatial near field and temporal profiles for the combined $2 \omega$ and $1 \omega$ tests. 


\subsection{Samples}

The samples investigated are fused silica, UV grade Corning 7980, 2-inch round 1-cm thick and were super polished by SESO. Laser damage was initiated off-line at $355 \mathrm{~nm}$ with a single shot at a fluence level in the 40 to $45 \mathrm{~J} / \mathrm{cm}^{2}$ range with a $7.5 \mathrm{nsec}$ FWHM Gaussian pulse. This high initiating fluence was chosen to produce repeatable damage spots in both size and morphology; even so there were variations in the site morphology. A typical initiated site is shown in figure 5. The sites typically contained multiple pits varying in individual sizes from 10 to $60 \mu \mathrm{m}$; some of these pits have a visible crack network associated with them and some pits are joined with adjacent pits.

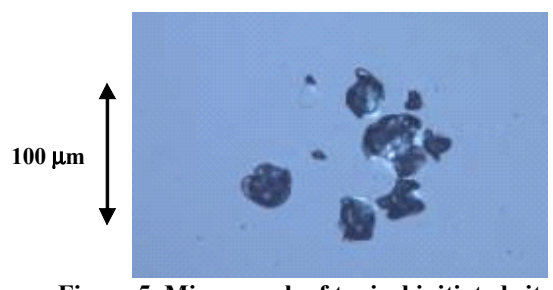

Figure 5. Micrograph of typical initiated site.

\section{EXPERIMENTAL RESULTS}

\subsection{Growth of exit surface damage with both $3 \omega$ and $1 \omega$ present.}

The goal of this work is to obtain the growth rate of laser damage on the exit surface under multiple wavelength irradiation. The result of all growth measurements obtained under conditions where the site was illuminated with both the third harmonic and the fundamental during the entire growth sequence is considered in this section. The site is allowed to grow until the diameter reaches two or more millimeters. At least 50 shots at the working fluences are accumulated before reporting that a site does not grow. In some cases as many as 300 shots were accumulated before reporting a zero growth site.

After each laser shot, the lateral area of the damaged site was measured. The lateral diameter of the damage was calculated from the measured area by assuming a circular equivalent area. A typical growth plot with the effective diameter plotted vs. shot number is shown in figure 6. Also, the average fluences in an area surrounding the growing site are plotted. In this case, the $3 \omega$ fluence was held at $5.2 \mathrm{~J} / \mathrm{cm}^{2}$ and the $1 \omega$ fluence was held at $7.0 \mathrm{~J} / \mathrm{cm}^{2}$. As was found for single wavelength growth, growth with two wavelengths simultaneously present exhibits a lateral diameter increase which is exponential with shot number. The data is fit to an exponential curve given by

$$
D=D_{0} e^{\alpha N}
$$

where $\mathrm{D}$ is the effective lateral diameter of the damage, $\mathrm{N}$ is the shot number and $\alpha$ is designated the growth coefficient. The lateral growth spurts seen in the plots are typified by a few shots where crack growth seen on the perimeter is followed by apparent spallation of material with this cycle repeating itself. The exponential fit to the data in figure 6 is shown along with the R-squared value for this fit. All of the sites tested show comparable fits to the data. 


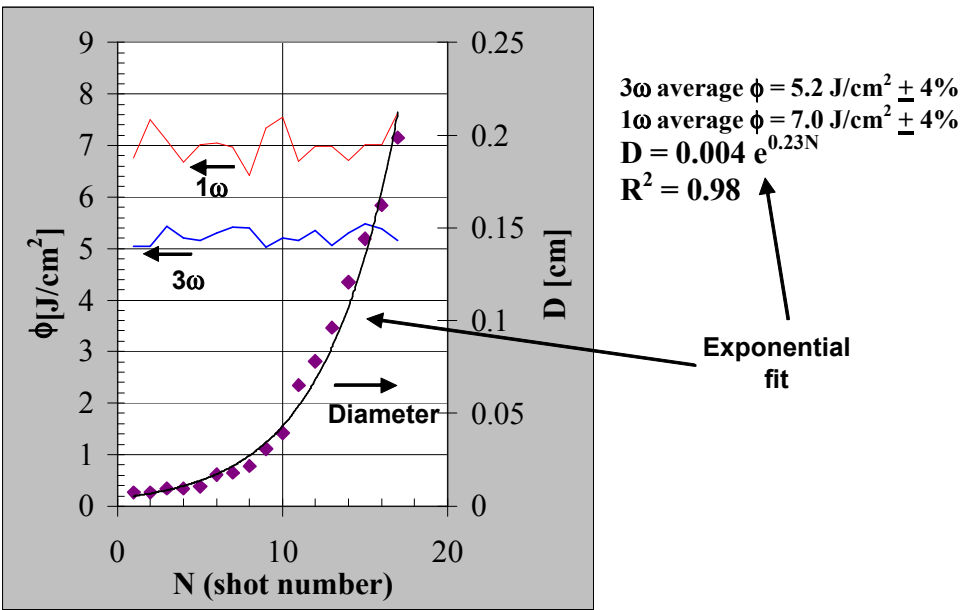

Figure 6. Typical lateral growth behavior showing exponential growth with shot number.

The growth behavior plotted in figure 6 was obtained from the transmitted laser beam during the growth sequence.

Plots of the growth coefficient vs. fluence for many sites shot with various pairs of fluences show a threshold behavior for growth as a function of the total fluence as can be seen in figure 7 where both the growth and no growth data are plotted. A linear fit to all the non-zero data is shown as the dark solid line.

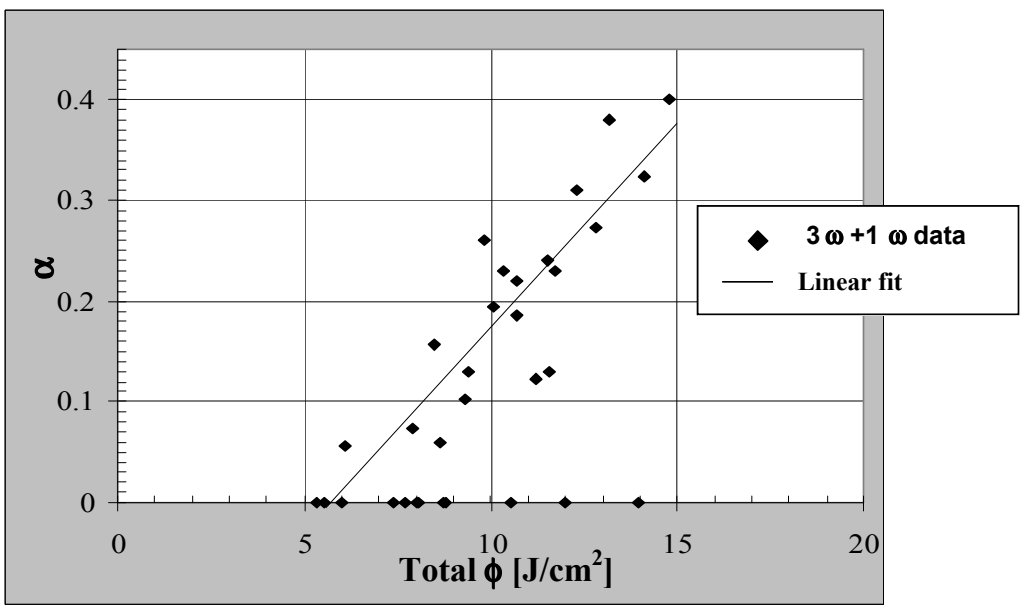

Figure 7. Growth coefficients for combined $1 \omega$ and $3 \omega$ vs. sum fluence.

The linear fit to the data, $\alpha=0.041 \mathrm{~F}-0.233$ with an $\mathrm{R}^{2}=0.7$, shown in figure 7 predicts a growth threshold of $5.7 \mathrm{~J} / \mathrm{cm}^{2}$; though some sites did not grow at fluences greater than this threshold. In figure 8 we have plotted all the sites that grew along with the growth data measured with only $3 \omega$ present. ${ }^{1}$ For this plot, the linear fit to the data obtained with $3 \omega$ only is given by $\alpha=0.030 \mathrm{~F}-0.193$ with an $\mathrm{R}^{2}=0.8$ which predicts a $16 \%$ lower growth threshold: $4.9 \mathrm{~J} / \mathrm{cm}^{2}$. The slope difference between the two linear fits of figures $7 \& 8$ is less that $5 \%$. With the exception of two sites the no growth sites in figure 7 were obtained when the $3 \omega$ component of the sum fluence was less than $4.9 \mathrm{~J} / \mathrm{cm}^{2}$. The data shown in figure 8 suggests that the growth characteristics are determined by the total fluence. 


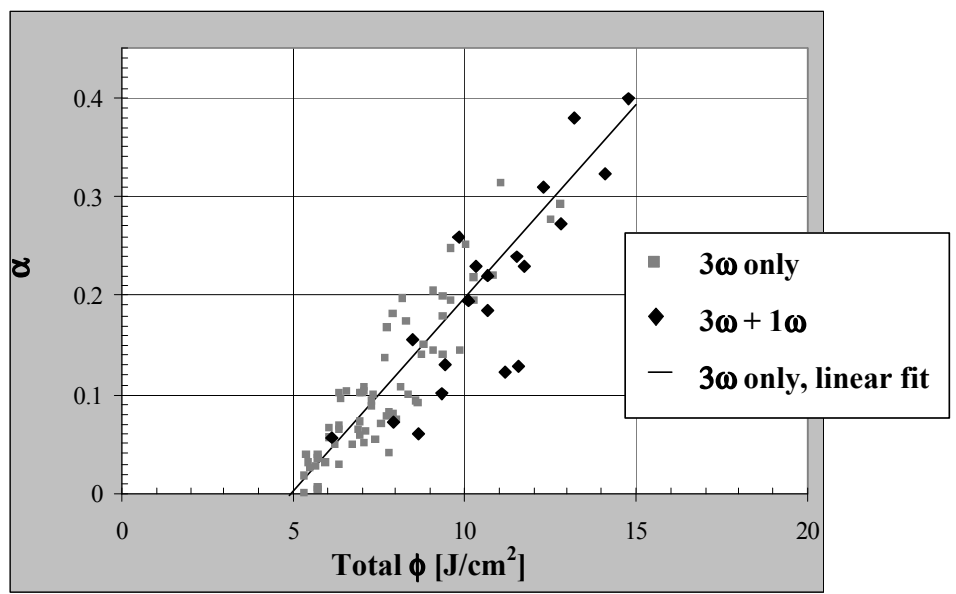

Figure 8. Plot of growth coefficients for $3 \omega$ only and $3 \omega$ plus $1 \omega$ vs. total fluence.

We have replotted the combined wavelength data by parameterizing the $3 \omega$ component of the total fluence; this is shown in figure 9 . This plot suggests that if the $3 \omega$ fluence is below the $3 \omega$ growth threshold then, the growth coefficient may be less than that which would have been measured if all the fluence had been delivered at $3 \omega$.

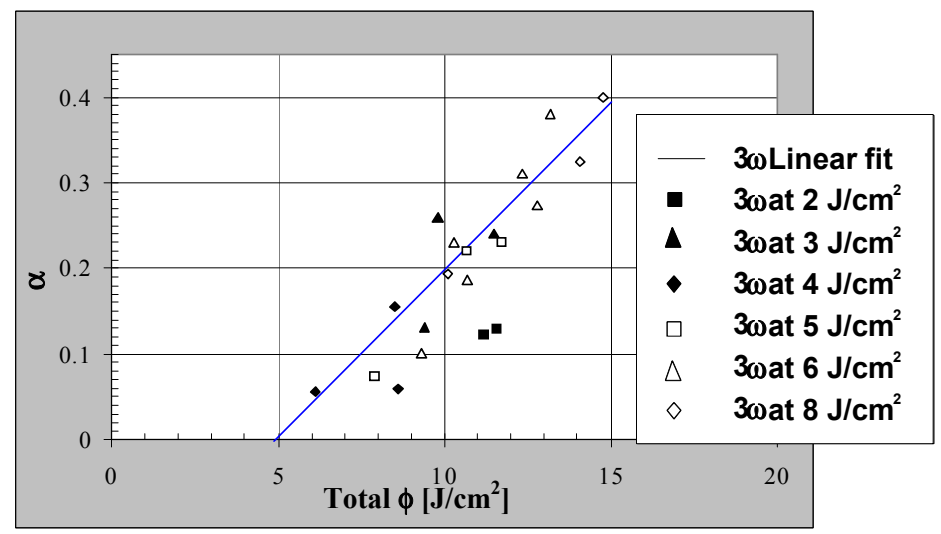

Figure 9. Combined $3 \omega$ and $1 \omega$ growth with $3 \omega$ component specified.

\subsection{Growth of exit surface damage sites with combined $2 \omega$ and $1 \omega$ irradiation.}

The growth coefficients previously obtained with $2 \omega$ only $^{2}$ are plotted versus fluence in figure 10 . Only sites having measurable growth are included on the plot. No growth was initiated at fluences below $12 \mathrm{~J} / \mathrm{cm}^{2}$, even on very heavily damaged sites. A linear fit to the measured data valid for fluences $\geq 12 \mathrm{~J} / \mathrm{cm}^{2}$ is given by $\alpha=0.0094 \mathrm{~F}-0.0423$ with an $\mathrm{R}^{2}=$ 0.8 is shown in figure 10 . Based on the observed $2 \omega$ threshold, $2 \omega$ fluences for the combined wavelength tests are chosen to be less than this threshold. 


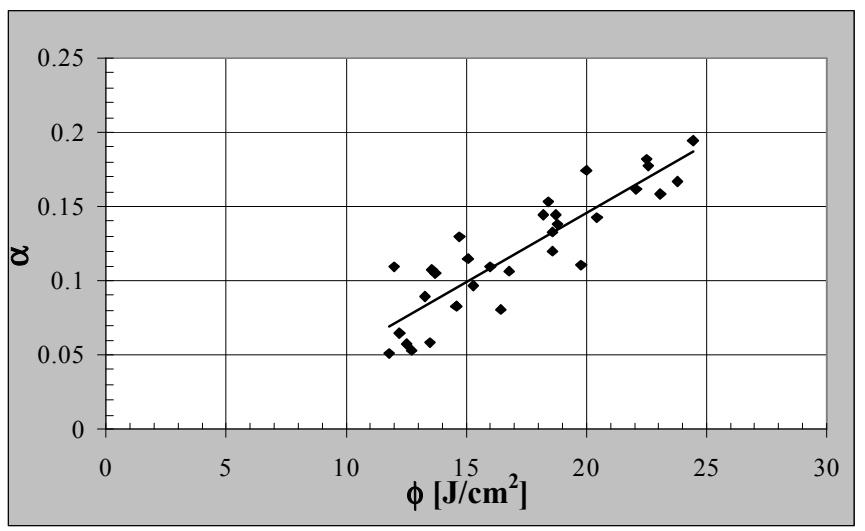

Figure 10. Growth coefficients for $2 \omega$ only.

The combined $2 \omega$ with $1 \omega$ growth test results are shown in figure 11 where growth coefficients for all sites including no growth sites are plotted versus the sum fluence and the data is parameterized with the $2 \omega$ fluence. Also included on the plot are both the $3 \omega$ and $2 \omega$ linear fits shown in figures 8 and 10 respectively.

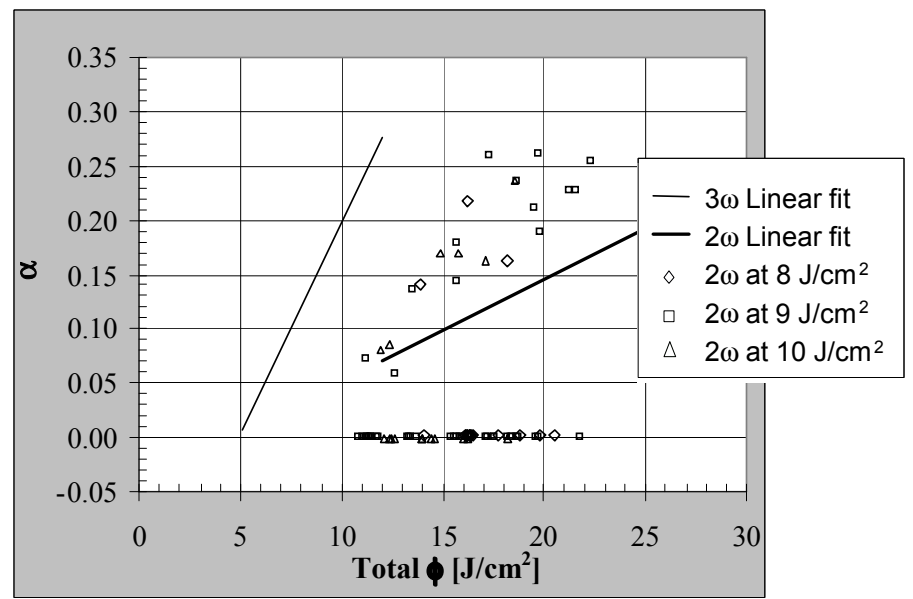

Figure 11. Growth coefficients for combined $2 \omega$ and $1 \omega$ vs. the total fluence.

In figure 12 , the non-zero growth data from figure 11 is shown along with non-zero growth coefficients obtained with $1 \omega$ only. ${ }^{3}$ As can be seen from this plot the combined fluence coefficients superimpose well with the single wavelength data obtained with $1 \omega$ only. 


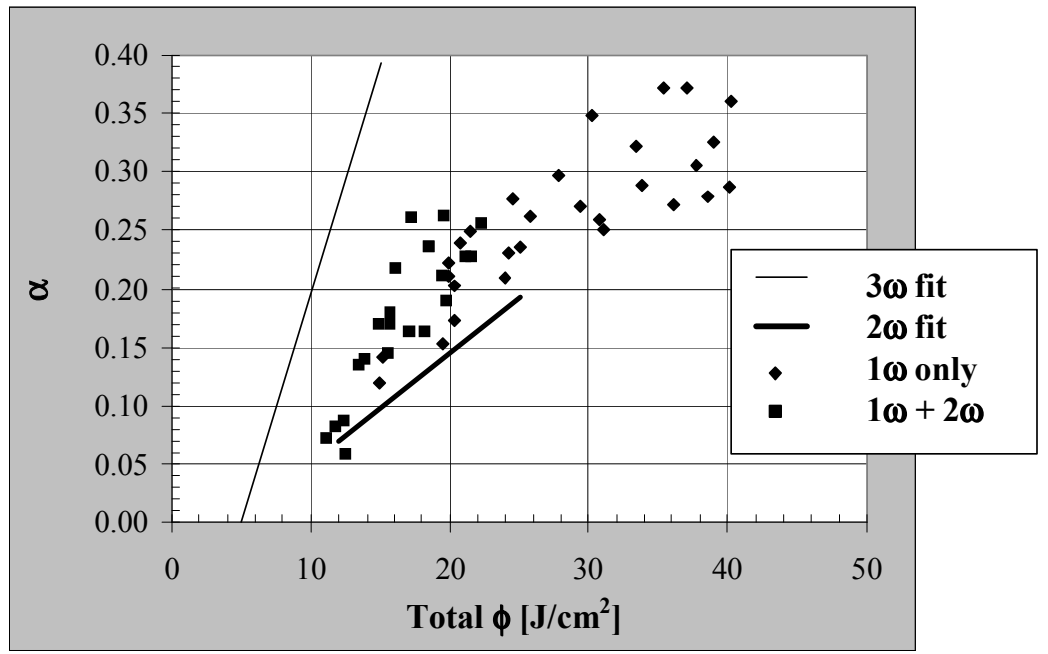

Figure 12. Growth coefficients for $1 \omega$ only along with $1 \omega+2 \omega$ growth data.

3.3 Summary of all wavelength exit surface growth.

All single wavelength data along with the multiple wavelength growth data have been combined into one plot shown in figure 13. Also shown on this plot is a second order fit to the $1 \omega$ only growth coefficients, given by $\alpha=-0.00036 \mathrm{~F}^{2}+0.028 \mathrm{~F}$ -0.21 with $\mathrm{R}^{2}=0.9$. This data fit, over the 15 to $40 \mathrm{~J} / \mathrm{cm}^{2}$ range where the data was obtained, seems to suggest a saturation effect in the growth coefficients; as of now we have been unable confirm this by measuring growth coefficients at fluences greater than $40 \mathrm{~J} / \mathrm{cm}^{2}$ as surface damage initiation dominates at these high fluences.

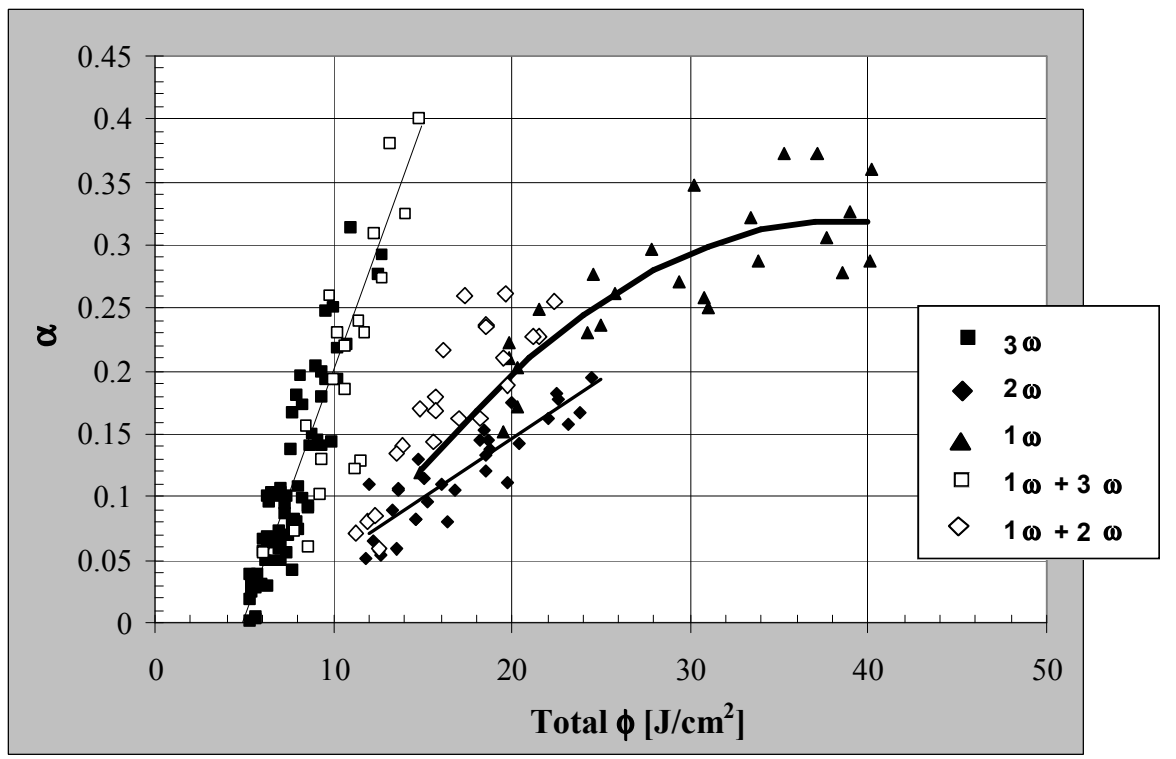

Figure 13. All non-zero growth data. 


\section{DISCUSSION}

We have shown that unconverted $1 \omega$ must be considered in the operations of frequency converted laser system when multiple wavelengths are present together on fused silica optical components. For both pairs of wavelengths, if a damage site grows it is the total absorbed energy that determines the damage growth rate. This is reasonable if we assume that absorption at one or both wavelengths leads to plasma generation during the growth event and that all subsequent incident laser energy is absorbed. For both pairs of wavelengths, the threshold for growth is not altered significantly from the single harmonic threshold provided that it is the total fluence that is considered. For the combined $3 \omega$ with $1 \omega$ case, once growth is enabled, the growth rate is similar to the $3 \omega$ only curve and the total energy determines the growth coefficient. This is in contrast to the combined $2 \omega$ with $1 \omega$ case, where again it is the total energy that determines the growth coefficient but now it corresponds to the $1 \omega$ only curve. In both cases, the measured coefficient corresponds to that at the wavelength having the greatest damage growth rate. The reason for this behavior is not yet explained. The fact that the observed single wavelength growth rates are not monotonic in wavelength needs to be understood as part of this eventual explanation

\section{ACKNOWLEDGEMENTS}

This work was performed under the auspices of the U. S. Department of Energy by University of California Lawrence Livermore National Laboratory under contract No. W-7405-Eng-48.

\section{REFERENCES}

1. M. A. Norton, L. W. Hrubesh, Z. Wu, E. E. Donohue, M. D. Feit, M. R. Kozlowski, D. Milam, K. P. Neeb, W. A. Molander, A. M. Rubenchik, W. D. Sell, and P. J. Wegner, "Growth of laser-initiated damage in fused silica at $351 \mathrm{~nm} "$ Proc. SPIE 4347, 468, 2000.

2. M. A. Norton, E. E. Donohue, W. G. Hollingsworth, J. N. McElroy, and R. P. Hackel, "Growth of laser-initiated damage in fused silica at $527 \mathrm{~nm} "$, Proc. SPIE 5273, 236, 2003.

3. M. A. Norton, E. E. Donohue, W. G. Hollingsworth, M. D. Feit, A. M. Rubenchik, and R. P. Hackel, "Growth of laserinitiated damage in fused silica at 1053 nm", Proc. SPIE 5647, 197, 2004.

4. C. B. Dane, L. E. Zapata. W. A. Neuman, M. A. Norton, and L. A. Hackel, "Design and Operation of a 150 W Near Diffraction-limited Laser Amplifier with SBS Wavefront Correction,” IEEE J. Quantum Electronics, 31, pp. 148-163, 1995. 